\title{
Comparación de tres métodos indirectos para estimar el crecimiento de la tilapia Oreochromis aureus (Perciformes: Cichlidae) en un lago tropical de México
}

\author{
Andrés Arellano-Torres, Daniel Hernández Montaño \& Carlos Meléndez Galicia \\ Centro Regional de Investigación Pesquera-Pátzcuaro, Instituto Nacional de Pesca- SAGARPA. Calzada Ibarra No. \\ 28, Col. Ibarra, Pátzcuaro, Michoacán, C. P. 61609, México; andres.arellano@inapesca.sagarpa.gob.mx, \\ daniel.hernandez@inapesca.sagarpa.gob.mx, carlos.melendez@inapesca.sagarpa.gob.mx
}

Recibido 13-VI-2012. Corregido 10-XI-2012. Aceptado 12-XII-2012.

\begin{abstract}
A comparative analysis of three length based methods for estimating growth of the tilapia Oreochromis aureus (Perciformes: Cichlidae) in a tropical lake of Mexico. Several methods are now available to estimate fish individual growth based upon the distribution of body lengths in a population. Comparative analyses of length-based methods have been undertaken mainly for marine species; nevertheless, limited information is available for inland species. Tilapia is one of the most important freshwater fisheries and its growth parameters have been estimated by several authors, usually using one length-based method. Thus, the main objectives of this study were: a) to estimate growth parameters of $O$. aureus from Chapala lake, Mexico, using three length-based methods ELEFAN, PROJMAT and SLCA; b) to quantify the effect of input data variations in growth parameters estimates by the jackknife technique; and c) to compare the new estimates with those previously reported, through the standard growth index $\phi^{\prime}$. We collected and analyzed a total of 1973 specimens from commercial landings from January to December 2010. The three length-base methods used in the present study resulted in parameter estimates within the range of those reported in other studies. Results derived from jackknife analysis revealed lowest values in the error percentage and coefficient of variation for $L_{\infty}$ when applying ELEFAN, while PROJMAT showed lowest values in the precision estimators for $\mathrm{K}$, which was very similar to ELEFAN. Estimates of the comparative growth index $\phi$ ' were also very similar to those reported for the same species when studied in different reservoirs. Considering our results, we suggest the use of ELEFAN rather than SLCA due to its accuracy to estimate growth parameters for O. aureus. Rev. Biol. Trop. 61 (3): 1301-1312. Epub 2013 September 01.
\end{abstract}

Key words: growth, length-based methods, jackknife, lake Chapala, Oreochromis aureus.

Las estimaciones de crecimiento son esenciales en el manejo de recursos pesqueros, ya que sirven como base en diversos modelos para describir y cuantificar la estimación de la mortalidad, reclutamiento, edad de primera madurez, rendimiento máximo sostenible entre otras variables poblacionales relacionadas a su explotación, con la finalidad de aplicar estrategias para un adecuado manejo y explotación (Isaac 1990, Haddon 2001). La estimación del crecimiento en recursos pesqueros puede realizarse mediante la aplicación de métodos directos e indirectos. Los métodos directos consisten en el registro de incrementos de talla en el tiempo mediante la lectura de marcas periódicas (anuales, estacionales o diarias) en estructuras rígidas como otolitos, escamas y vértebras entre otros. Sin embargo, su aplicación genera altos costos económicos y requiere largos tiempos para la obtención y procesamiento de las muestras (Sparre et al. 1989); aunado al hecho de que en algunos organismos acuáticos (por ejemplo, crustáceos) es difícil realizar estimaciones de edad y crecimiento con estos métodos (Isaac 1990). Por otro lado, los métodos indirectos basados en 
el análisis de frecuencias de tallas son alternativas comúnmente utilizadas para estimar el crecimiento, debido a que los datos de longitud son relativamente sencillos de obtener, el tiempo de procesamiento es rápido e implica bajos costos de operación (Sparre et al. 1989, Isaac 1990). Sin embargo, el uso generalizado de estos métodos está limitado por las características de las distribuciones de frecuencia de tallas utilizadas y su compatibilidad con los algoritmos que lo sustentan. Por lo que, para cada aplicación particular, se debe conocer los supuestos de los métodos aplicados, sus algoritmos y las características del ciclo de vida y biología de la población. De esta manera, la elección del método apropiado para estimar el crecimiento dependerá de las características particulares de la población estudiada (Galindo 2005). Para estimar el crecimiento, actualmente existen diversos métodos basados en la distribución de tallas. Tres de los métodos indirectos más populares son: 1) ELEFAN (Electronic Length Frequency Analysis) (Gayanilo et al. 1994), 2) SLCA (Sheperd's Length Composition Analysis) (Sheperd 1987b) y 3) PROJMAT (Projection Matrix Method) (Rosenberg et al. 1986, Sheperd 1987a). La evaluación de la aplicación de estos métodos indirectos para estimar el crecimiento, se han realizado esencialmente en especies de origen marino (Defeo et al. 1992a, Leonce-Valencia \& Defeo 1997, Nepita-Villanueva \& Defeo 2001, Galindo 2005). Sin embargo, no se ha realizado un análisis comparativo de la aplicación de estos métodos en especies de aguas continentales; a pesar de que en los últimos años la pesca en aguas interiores tiene gran importancia, debido a la creciente demanda nutricional por la obtención de alimento de alto contenido proteico y por la creación de fuentes de empleo (BeltránÁlvarez et al. 2010).

Una de las pesquerías de aguas continentales más importantes en México es la pesca de tilapia, su producción en el año 2010 fue de 81 250ton, lo que representa el 5.02\% de la producción pesquera nacional (CONAPESCA 2010). La pesca de especies como la tilapia (Oreochromis spp.), ha generado importantes beneficios sociales y económicos, principalmente para las comunidades rurales relacionadas con la pesquería, debido a que se caracteriza por presentar un rápido crecimiento, una fácil comercialización, además de que es considerada como un producto altamente nutritivo, relativamente barato y de fácil adquisición (Jiménez-Badillo 2006). En el lago de Chapala, la tilapia es una de las especies más importantes en la captura comercial. En 2003 representó el $42 \%$ de la producción total del lago (Diario Oficial de la Federación 2006) y en 2007 el 51.5\% (C. Meléndez en prep.); sin embargo sus volúmenes de captura muestran signos de sobrexplotación del recurso (HernándezMontaño 2006), por lo cual es necesario proporcionar alternativas que sirvan de base para una adecuada administración de este recurso, así como para reforzar los lineamientos de las investigaciones al respecto.

Para estimar el crecimiento de tilapia se han realizado diversos estudios (Bernal 1984, Ramírez 2007, Beltrán-Álvarez et al. 2010, Gómez-Ponce et al. 2011), algunos de ellos se han basado en métodos indirectos (RamosCruz 1995, Jiménez-Badillo 2006, HernándezMontaño 2006, Gómez-Márquez et al. 2008, Peña-Messina et al. 2010). Sin embargo, en la mayoría de los estudios previos no se han realizado estimaciones alternativas por más de un método, ni tampoco se ha cuantificado la incertidumbre asociada a dichas estimaciones. Esta incertidumbre cobra especial importancia en recursos tropicales como la tilapia (Oreochromis spp), donde ciertas características del ciclo de vida (e. g., ciclo reproductivo y reclutamientos continuos) impiden una clara progresión de clases modales, lo cual resalta la importancia de determinar la influencia que tiene cada grupo de datos mensuales en la estimación de los parámetros de crecimiento (Leonce-Valencia \& Defeo 1997). Es muy común además la presencia de errores de medición y sesgos en la obtención de datos, por lo cual es necesario evaluar la precisión de cada método.

En este contexto, los objetivos del presente trabajo fueron: a) estimar los parámetros de crecimiento de $O$. aureus del lago de 
Chapala, usando los métodos indirectos de ELEFAN, SLCA y PROJMAT; b) cuantificar la incertidumbre en los parámetros del modelo, resultantes de variaciones en la estructura de la base de datos y c) comparar las estimaciones con aquellas reportadas en la literatura para $O$. aureus, usando el índice de crecimiento estándar ф’ (Pauly \& Munro 1984).

\section{MATERIALES Y MÉTODOS}

Área de estudio: El lago de Chapala es un cuerpo de agua natural que por su tamaño es considerado el más grande de México y el tercero en Latinoamérica (Guzmán-Arroyo 1995). Se localiza en la meseta central en los estados de Jalisco y Michoacán, entre los $20^{\circ} 07^{\prime} 00^{\prime \prime}$ - 202' $30^{\prime \prime} \mathrm{N}$ y $102^{\circ} 40^{\prime} 45^{\prime \prime}$ - $103^{\circ} 25^{\prime} 30^{\prime \prime} \mathrm{W}$. En la parte oriental recibe aguas de los Ríos Lerma y Duero, en la parte sur de los Ríos Jiquilpan, de la Pasión y Sahuayo, y al norte del Río Zula, en la parte noreste tiene como su principal afluente el Río Santiago. Presenta una superficie de $1100 \mathrm{~km}^{2}$, tiene una profundidad media de $4.5 \mathrm{~m}$ con una máxima de $7 \mathrm{~m}$ (Hernández-Montaño 2006). En el lago de Chapala, la dinámica hidrológica, está definida por la entrada del Río Lerma. El contenido de oxígeno disuelto es alto en todo el cuerpo de agua $(>5.0 \mathrm{mg} / \mathrm{L})$ debido a la constante circulación durante todo el año. Estos perfiles de oxígenos así como los de temperatura permiten clasificar a la laguna como polimíctico cálido continuo. El contenido de fósforo es muy alto, lo que se refleja en elevados contenidos de clorofila (a), por lo que se le puede clasificar como eutrófico (E. Arredondo, en prep.).

Los ejemplares de $O$. aureus se obtuvieron de doce muestreos de la captura comercial, de enero a diciembre de 2010 en el lago de Chapala. Con la finalidad de garantizar la representatividad del espectro de tallas y sexos, se examinaron especímenes en diferentes localidades pesqueras del lago y para disminuir el efecto de la selectividad del arte de pesca, los organismos se obtuvieron de redes agalleras con aberturas de malla desde 2" hasta 5", que miden entre 60 y $150 \mathrm{~m}$ de largo por 1.5 a $2 \mathrm{~m}$ de alto. Para maniobrarlas, se tienden en el agua y se fijan de forma que ésta se extienda lo suficiente para que los organismos logren atravesar y se enmallen en ella, las redes comúnmente operan día y noche.

Los organismos se identificaron mediante el uso de claves dicotómicas (Trewavas 1983, Arredondo-Figueroa \& Guzmán-Arroyo 1986, Arredondo-Figueroa \& Tejeda-Salinas 1989) y a cada uno se les registró la longitud patrón (LP; $\pm 0.1 \mathrm{~cm})$ que comprendió desde la punta de la cabeza al pedúnculo caudal, utilizando un ictiómetro convencional, así como el peso total ( $\mathrm{PT} ; \pm 0.1 \mathrm{~g})$. El sexo se registró mediante la observación directa de las gónadas (Morales 1991). La relación longitud-peso fue descrita de acuerdo a Ricker (1975) y se utilizó la prueba-t de student para determinar si los peces muestran diferencias respecto a un crecimiento isométrico $(b=3)$ (Pauly 1984). Las frecuencias de tallas fueron agrupadas en un intervalo de clase de $1 \mathrm{~cm}$, siguiendo criterios de Defeo et al. (1992b).

Para estimar los parámetros de crecimiento se aplicaron los métodos indirectos ELEFAN (Gayanilo et al.1994), SLCA (Sheperd 1987b) y PROJMAT (Rosenberg et al. 1986, Sheperd 1987a), que asumen un crecimiento individual descrito por el modelo de von Bertalanffy (Defeo et al. 1992a). En resumen cada método funciona de la siguiente manera:

1. ELEFAN I: identifica picos y valles en las distribuciones de frecuencia de tallas y ajusta una curva de crecimiento la cual pasa por el máximo número de picos, sin suponer normalidad en dichas distribuciones. Un índice de bondad de ajuste denominado $R n$ (ver Gayanilo et al. 1994 para detalles) es determinado por la forma exponencial de la relación ESP/ASP, donde ESP representa la Suma Explicada de Picos y ASP la Suma Disponible de los Picos como:

$$
\mathrm{Rn}=\frac{10^{\mathrm{ESP} / \mathrm{ASP}}}{10}
$$


2. SLCA: estima el crecimiento basado en una función coseno, la cual es independiente del número y posición de las modas (Shepherd 1987a). Esta función es expresada como:

$$
T(l)=\frac{\operatorname{sen} \pi\left(t_{\max }-t_{\min }\right)}{\pi\left(t_{\max }-t_{\min }\right)} \cos 2 \pi\left(t_{\text {prom }}-t_{s}\right)
$$

Donde $t_{\max } \mathrm{y} t_{\min }$ son las edades correspondientes a los limites superior e inferior del respectivo intervalo de longitud, $t_{\text {prom }}$ es la edad promedio y $t_{s}$ es la fracción del año en la cual la muestra fue recolectada (Shepherd 1987a). Una función de prueba, la cual es positiva para modas y negativa entre modas, calcula una puntuación derivada de la suma de todas las categorías de longitud, siendo maximizada por la combinación más adecuada de parámetros de crecimiento. La función de prueba de la puntuación está dada por:

$$
\mathrm{S}=\sum_{l} \sum_{i}(l, i) N^{0.5}(l, i)
$$

Donde $S$ es la función de puntaje, $l$ hace referencia a los grupos de longitud respectivos, $i$ a las distribuciones identificadas (disponibles) y $N$ son las frecuencias observadas (Jones et al. 1990).

3. PROJMAT: está basado en la matriz poblacional de proyección de Leslie (Leslie 1945). Shepherd (1987b) mostró que una matriz de transición $(A)$ puede ser resuelta directamente como:

$$
\vec{N}(t+1)=A \vec{N}(t)
$$

Donde $\vec{N}(t)$ y $\vec{N}(t+1)$ son vectores que describen la estructura de la población en el tiempo $t$ y $t+1$ respectivamente. Puede ser desagregada como:

$$
A(j, i)=G(j, i) S(i)
$$

Donde $G(j, i)$ es la matriz de crecimiento, sus elementos son proporciones de cada grupo de longitud inicial $i$ que contribuye a formar el grupo de longitudes finales $j$ asumiendo la ecuación de crecimiento de von Bertalanffy, $S(i)$ es la sobrevivencia promedio del intervalo $i$; con éstos elementos PROJMAT proyecta la muestra obtenida del tiempo $t$ al tiempo $t+1$, usando valores semilla de $\mathrm{K}$ y $L_{\infty}$. La distribución de frecuencia de longitud proyectada es comparada estadísticamente con la observada a través de la suma de diferencias cuadráticas (SSQ). Este procedimiento es repetido hasta que se llevan a cabo todas las comparaciones entre $n$ muestras para un rango amplio de valores de $\mathrm{K}$ y $L_{\infty}$, donde las mejores estimaciones son aquellas que minimizan la SSQ (Basson et al. 1988).

Para evaluar la influencia que tiene cada muestra por mes en la estimación de los parámetros de crecimiento, se aplicó la técnica de corte jackknife (Efron 1982, Levi et al. 1987), la cual consiste en suprimir cada uno de los 12 meses secuencialmente con sustitución, generando 12 grupos de 11 datos por mes. Por lo tanto se obtuvieron 12 combinaciones diferentes de parámetros de crecimiento. Se utilizaron el error porcentual (EP) y el coeficiente de variación porcentual $(\mathrm{CV})$ como estimadores de precisión. El primero fue obtenido como:

$$
\mathrm{EP}=\frac{(S t-S t j) * 100}{S t j}
$$

Donde $S t$ es el parámetro de crecimiento estimado usando la muestra total (12 meses) y $S t_{j}$ es el promedio del parámetro estimado derivado de los $n$ pseudovalores jackknife. EP es óptimo cuando es igual a cero. El CV jackknife fue obtenido por:

$$
C V=\frac{\sqrt{\sum\left(S t_{i-1}-S t_{j}\right)^{2} / n(n-1)}}{S t j} * 100
$$

Donde $\mathrm{St}_{\mathrm{i}-1}$ es el estimador del parámetro al omitir una muestra y $n$ es el tamaño de la muestra anual $(n=12)$.

El índice de crecimiento estándar ( $\phi ')$ (Pauly \& Munro 1984) fue usado como criterio para comparar las diferentes estimaciones de crecimiento, el cual fue aplicado para cada par de valores $\mathrm{K}$ y $L_{\infty}$ obtenidos con todos los datos 
por cada método y por los valores obtenidos del análisis de jackknife. La ecuación que define este índice es:

$$
\phi^{\prime}=2 \log 10\left(\mathrm{~L}_{\infty}\right)+\log 10 \mathrm{~K}
$$

Este criterio fue elegido debido a que proporciona un parámetro unificado del crecimiento, y aunque se deriva de $\mathrm{K}$ y de $L_{\infty}$, los valores de $\phi$ ' no muestran grandes oscilaciones como los que se presentan con los parámetros clásicos (Sparre et al. 1989).

\section{RESULTADOS}

La población estudiada consistió en un total de 1973 organismos de $O$. aureus, 1012 fueron hembras $(51.3 \%)$ y 961 fueron machos (48.7\%). Las tallas estuvieron en una escala de 10 a $35 \mathrm{~cm}$ de LP (Fig. 1) y los pesos variaron de 30 a $700 \mathrm{~g}$ de PT. La relación de sexos macho: hembra no fue estadísticamente diferente de $1: 1\left(\chi^{2}=0.02, p>0.05\right)$, por lo que hembras y machos fueron agrupados de forma general para su análisis. El mejor modelo que describió la relación longitud-peso (LP vs PT) fue de tipo potencial, el cual se expresó como: $\mathrm{PT}=0.104(\mathrm{LP})^{2.62}\left(\mathrm{r}^{2}=0.84, \mathrm{p}<0.05\right)$. El valor del exponente $(b=2.62)$, fue significativamente diferente de tres (prueba-t, $\mathrm{p}<0.05$ ), por lo que el crecimiento de la especie en este embalse se consideró como alométrico.

Conjunto completo de datos: $\mathrm{Al}$ considerar toda la información (los 12 meses de muestreo), los resultados obtenidos por los tres métodos indirectos estimaron valores similares de $L_{\infty}$, los cuales oscilaron de 38 a $40 \mathrm{~cm}$ de $\mathrm{LP}$, mientras que los valores $\mathrm{K}$ fueron de 0.30 a $0.35 /$ año, para $\mathrm{t}_{0}$ los valores fueron de -0.18 a -0.56años, es decir mostraron una amplia diferencia entre métodos (Cuadro 1). SLCA y PROJMAT presentaron múltiples picos de convergencia.

Análisis jackknife: La iteración del análisis jackknife que omite secuencialmente uno de los 12 meses de muestreo, mostraron diferentes efectos de las variaciones aleatorias en los datos de entrada entre los tres métodos indirectos utilizados (Fig. 2). En el caso de ELEFAN, $L_{\infty}$ varió de 33.7 a $45 \mathrm{~cm}$ de LP, mientras que $\mathrm{K}$ estuvo en el intervalo de 0.26 a 0.60 /año; mostró una progresión negativa, la más alta $L_{\infty}$ y la más baja $\mathrm{K}$ ocurrieron cuando enero fue omitido, mientras que los valores más bajos de $L_{\infty}$ y los más altos de $\mathrm{K}$ resultaron cuando febrero fue omitido (Fig. 2A). La aplicación jackknife con SLCA mostró menos variación en las estimaciones de $L_{\infty}(42.8-45.7 \mathrm{~cm}$ de LP) y presentó moderada variabilidad para $\mathrm{K}$ (0.30-0.60/año). Sin embargo, a pesar de que los resultados mostraron poca variabilidad, no hubo una relación predecible entre $L_{\infty} \mathrm{y} \mathrm{K}$, cuando se omitió abril se presentaron los valores más altos que para los otros once conjunto de datos (Fig. 2B). PROJMAT presentó las mayores variaciones en las estimaciones de $L_{\infty}$ (30-43cm de LP) y la menor variabilidad en los resultados de K (0.25-0.45/año) (Fig. 2C).

Los estimadores de precisión EP y CV derivados del análisis jackknife, mostraron que ELEFAN consistentemente presentó los valores más bajos de EP (0.67\%) y CV (2.15\%) para $L_{\infty}$; PROJMAT registró los valores mínimos de EP (6.97\%) y CV (5.13\%) para K; en tanto que SLCA mostró los valores máximos de EP y $\mathrm{CV}$ en los tres parámetros de crecimiento (Cuadro 1).

Comparaciones con el índice de crecimiento estándar $\phi^{\prime}$ : El análisis comparativo de $\phi$ ' derivado de la omisión secuencial de muestras fue similar entre los valores estimados por ELEFAN y SLCA, mientras que PROJMAT generó los valores más bajos. La figura 3 muestra la variación de los valores de $\mathrm{K}$ y $L_{\infty}$ generados del análisis jackknife. De la omisión secuencial de una muestra con ELEFAN se estimaron valores de $\phi$ ' entre 2.63 (cuando enero fue omitido) y 2.97 (cuando diciembre fue omitido). SLCA presentó valores de $\phi$ ' en el rango de 2.74 (cuando junio fue omitido) a 3.16 (cuando abril fue omitido). PROJMAT presentó el valor mínimo (2.43) cuando diciembre fue 


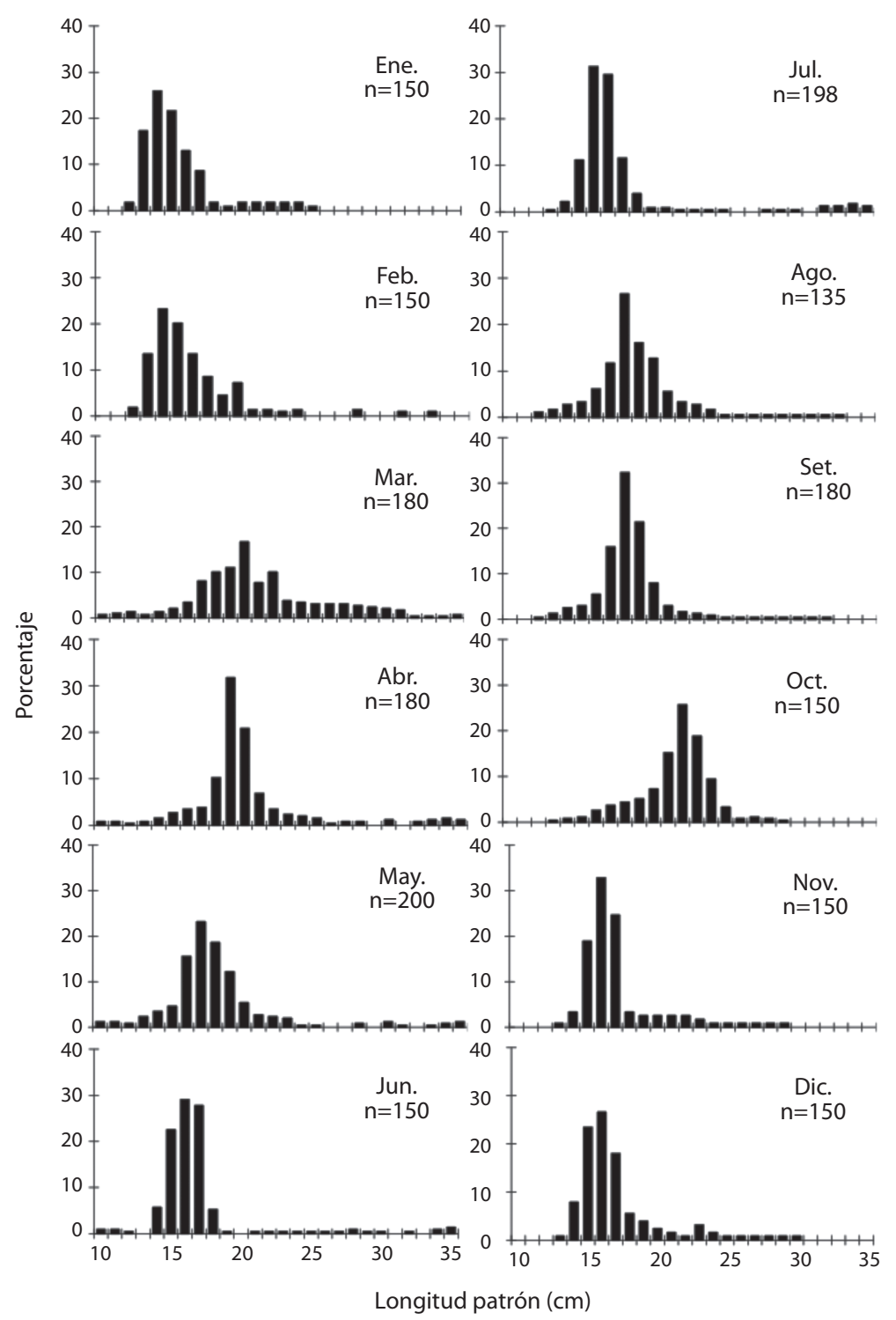

Fig. 1. Distribución de frecuencias de longitud mensual de Oreochromis aureus en el lago de Chapala. Fig. 1. Monthly length frequency distributions of Oreochromis aureus from lake Chapala.

omitido y el dato más alto (2.77) cuando julio fue omitido (Fig. 3).

\section{DISCUSIÓN}

En el presente estudio las tallas de $O$. aureus estuvieron en una escala de 10 a $35 \mathrm{~cm} \mathrm{LP}$, las tallas mínimas fueron similares a las reportadas para la presa Aguamilpa (10 a $40.3 \mathrm{~cm}$ LP) en el estado de Nayarit (Peña-Messina et al. 2010), sin embargo, fueron mucho mayores a las registradas por Gómez-Ponce et al. (2011) en la presa Zimapán (3.8 a $23.2 \mathrm{~cm}$ LP). Esto puede estar relacionado a las diferencias que hay entre las artes de pesca usadas en el lago de Chapala, con respecto a los otros cuerpos 


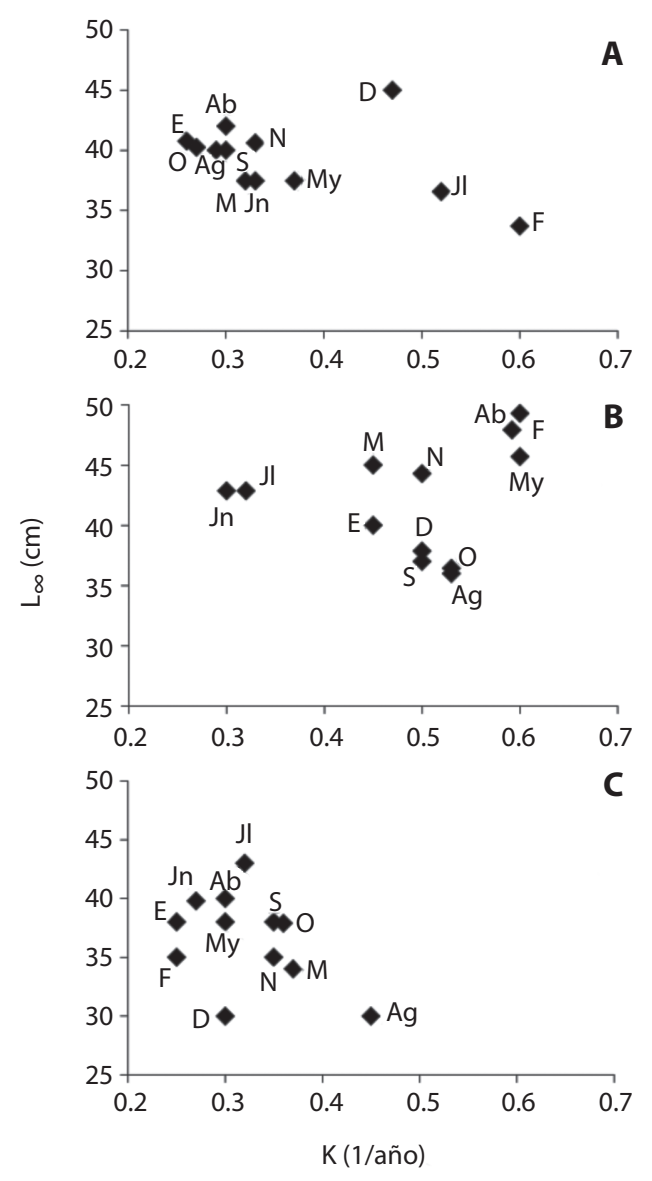

Fig. 2. Relación entre $L_{\infty}$ y K para Oreochromis aureus en el lago de Chapala, estimados por (A) ELEFAN, (B) SLCA y (C) PROJMAT. Cada letra se refiere al mes omitido en el análisis jackknife.

Fig. 2. Relationship between $L_{\infty}$ and $\mathrm{K}$ in Oreochromis aureus from lake Chapala, estimated by (A) ELEFAN, (B) SLCA and (C) PROJMAT. Each letter refers to the month omitted in jackknife analysis.

de agua. El análisis de la relación longitudpeso (LP vs PT) mostró un crecimiento de tipo alométrico. Este mismo tipo de crecimiento ha sido reportado para $O$. aureus (Peña-Messina et al. 2010) y para $O$. niloticus (Gómez-Márquez et al. 2008). Estudios realizados en distintas especies de tilapias y cuerpos de agua en México, muestran que los valores de la pendiente de esta relación oscila entre 2.5 y 3.5 (Granado 1996). Esto indica que el valor de $b=2.62$ se encuentra dentro del intervalo mencionado, y

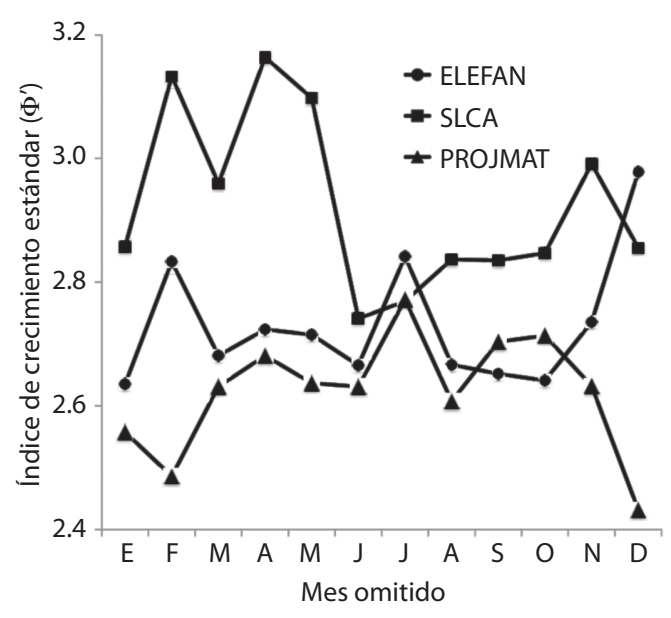

Fig. 3. Variación mensual de $\phi$ ' para Oreochromis aureus obtenidas de las estimaciones de $L_{\infty}$ y K en conjunción con el análisis de jackknife.

Fig. 3. Monthly variations of ' ${ }^{\prime}$ in Oreochromis aureus obtained from estimates of $L_{\infty}$ and $\mathrm{K}$ in conjunction with the jackknife analysis.

da confiabilidad a los resultados obtenidos en este trabajo para las tilapias del lago de Chapala. Por otra parte, el crecimiento alométrico para diferentes especies de tilapia ha sido atribuido principalmente a problemas de alimento y densidades en el agua (Murphy et al. 1991)

Respecto a los tres métodos indirectos evaluados en el presente estudio, ELEFAN mostró los mejores valores (más bajos EP y CV), seguido de PROJMAT y posteriormente SLCA. Lo anterior se basa a partir: 1) de una comparación de los parámetros de crecimiento $L_{\infty} \mathrm{y} \mathrm{K}$ y del índice de crecimiento estándar $\phi$ ' estimados por los tres métodos indirectos con los resultados previamente reportados en la literatura, que hacen estimaciones con métodos directos; 2) de los dos estimadores de precisión EP y CV derivados del análisis jackknife y 3) de evaluar la incertidumbre de los parámetros de crecimiento resultantes de variaciones en la base de datos por medio de la técnica de jackknife.

En cuanto a la comparación de los parámetros de crecimiento calculados con los tres métodos indirectos utilizados en este trabajo, se observó que los valores de $L_{\infty}$ y K son semejantes a los reportados para $O$. aureus por Bernal 
CUADRO 1

Parámetros de crecimiento de O. aureus en el lago de Chapala, estimados por ELEFAN, SLCA y PROJMAT, $\sin (\mathrm{St}) \mathrm{y}$ con $(\mathrm{P})$ jackknife. Se presentan los estimadores de precisión

TABLE 1

Growth parameters of $O$. aureus from lake Chapala, estimated by ELEFAN, SLCA and PROJMAT, without (St) and with (P) jackknife. Precision estimates are also presented

\begin{tabular}{|c|c|c|c|c|}
\hline \multirow{2}{*}{ Parámetros } & & \multicolumn{3}{|c|}{ Métodos } \\
\hline & & ELEFAN & SLCA & PROJMAT \\
\hline \multirow[t]{4}{*}{$L_{\infty}(\mathrm{cm})$} & St & 39.00 & 40.00 & 38.00 \\
\hline & $\mathrm{P}$ & 39.20 & 42.10 & 36.50 \\
\hline & EP (\%) & 0.67 & 4.98 & 3.94 \\
\hline & CV $(\%)$ & 2.15 & 3.13 & 3.09 \\
\hline \multirow{4}{*}{ K (1/año) } & St & 0.31 & 0.35 & 0.30 \\
\hline & $\mathrm{P}$ & 0.36 & 0.48 & 0.32 \\
\hline & EP $(\%)$ & 14.67 & 28.4 & 6.97 \\
\hline & $\mathrm{CV}(\%)$ & 8.59 & 5.79 & 5.13 \\
\hline \multirow[t]{4}{*}{$\mathrm{t}_{\mathrm{o}}$ (años) } & St & & -0.18 & -0.56 \\
\hline & $P$ & & -0.61 & -0.44 \\
\hline & EP $(\%)$ & & 70.50 & 26.19 \\
\hline & CV (\%) & & 12.80 & 11.58 \\
\hline Bondad de ajuste * & & $\mathrm{Rn}=0.228$ & $\mathrm{~S}=45.080$ & $\mathrm{SSQ}=0.396$ \\
\hline
\end{tabular}

*El índice de bondad de ajuste para ELEFAN (Rn), para SLCA (S) y la suma de cuadrados (SSQ) para PROJMAT se muestran.

*Goodness of fit for ELEFAN (Rn), for SLCA (S) and the sum of squared differences (SSQ) for PROJMAT are shown.

(1984), Ramírez (2007), Beltrán-Álvarez et al. (2010), Gómez-Ponce et al. (2011); quienes estimaron los parámetros de crecimiento a través de métodos directos (Cuadro 2).

El análisis jackknife permitió determinar una fuerte variabilidad en los parámetros individuales de crecimiento estimados por los tres métodos, en especial para SLCA. ELEFAN presentó la menor variabilidad seguida de PROJMAT, con valores dentro de los rangos reportados previamente en la literatura. El análisis comparativo del índice de crecimiento $\phi$ ' en conjunción con jackknife: corroboró la gran utilidad de éste parámetro a efectos comparativos. Los valores de $\phi$ ' obtenidos con SLCA, fueron ligeramente más altos a los obtenidos con ELEFAN y PROJMAT. Sin embargo, los parámetros de crecimiento estimados por medio de métodos directos e indirectos en otros estudios, se compararon con los resultados obtenidos por los tres métodos indirectos utilizados en la presente investigación. Los valores del índice de crecimiento $\phi$ ', calculados con $L_{\infty}$ y $\mathrm{K}$ reportados en estudios previos, variaron entre 2.29 y 3.02, intervalo que oscila dentro de los valores de $\phi$ ' obtenidos por ELEFAN, SLCA y PROJMAT en este estudio.

Con base en las características biológicas del recurso y de acuerdo a los resultados de los tres métodos indirectos evaluados en el presente estudio, ELEFAN fue el más adecuado para estimar los parámetros de crecimiento de $O$. aureus, debido a que mostró los valores mínimos en los estimadores de precisión (EP y $\mathrm{CV}$ ) para $L_{\infty}$, un valor similar de $\mathrm{K}$ al estimado por PROJMAT, quien en éste caso presentó los valores mínimos de EP y CV. Sin embargo, Jiménez-Badillo (2006) que estimó el crecimiento de $O$. aureus con ELEFAN, SLCA Y PROJMAT; sugiere que el método de ELEFAN 
CUADRO 2

Parámetros de crecimiento para O. aureus reportados en la literatura

TABLE 2

Growth parameters for $O$. aureus reported in the literature

\begin{tabular}{llcccl}
\multicolumn{1}{c}{ Embalse } & \multicolumn{1}{c}{ Método } & $* L_{\infty}(\mathrm{cm})$ & $\mathrm{K}(1 /$ año $)$ & $\phi^{\prime}$ & \multicolumn{1}{c}{ Fuente } \\
Presa Infiernillo, Michoacán & Escamas & 41.9 & 0.11 & 2.29 & Bernal (1984) \\
Presa Benito Juárez, Oaxaca & Battacharya & 29.6 & 0.21 & 2.26 & Ramos-Cruz (1995) \\
Presa Infiernillo, Michoacán & Escamas y opérculos & 47.9 & 0.46 & 3.02 & Jiménez-Badillo (2006) \\
& ELEFAN & & & & \\
& PROJMAT & 60.0 & 0.22 & 2.90 & \\
& SLCA & 39.6 & 1.03 & 3.21 & \\
Lago de Chapala, Jalisco-Michoacán & ELEFAN & 28.4 & 0.38 & 2.49 & \\
Presa Adolfo López Mateos, Sinaloa & Escamas & 41.6 & 0.37 & 2.80 & Hernández-Montaño (2006) \\
Presa Sanalona, Sinaloa & Escamas & 39.0 & 0.43 & 2.81 & Ramírez (2007) \\
Presa Aguamilpa, Nayarit & ELEFAN & 41.6 & 0.36 & 2.80 & Beltrán-Álvarez et al. (2010) \\
Presa Zimapan, Hidalgo & Escamas & 43.3 & 0.36 & 2.83 & Peña-Messina et al. (2010) \\
Lago de Chapala, Jalisco-Michoacán & ELEFAN & 28.1 & 0.33 & 2.41 & Gómez-Ponce et al. (2011) \\
& SLCA & 39.0 & 0.31 & 2.67 & Este trabajo \\
& PROJMAT & 40.0 & 0.35 & 2.74 &
\end{tabular}

$* L_{\infty}=$ Longitud patrón. $/ * L_{\infty}=$ Standard length.

es el menos adecuado para evaluar el crecimiento de tilapia, y debido a que es sensible a las modas discretas en los datos frecuencia de tallas, es importante mencionar que en ese estudio no se evaluó la variabilidad en los parámetros individuales de crecimiento estimados por los tres métodos y cabe señalar que en el estudio mencionado, a pesar de que se detectaron 11 grupos de edad mediante opérculos y escamas, los parámetros de crecimiento fueron sobrestimados por ELEFAN. No obstante, se ha mostrado que ELEFAN trabaja mejor cuando se incluyen más grupos de edad (Defeo et al. 1992a). Además menciona que PROJMAT proporciona una estimación más fiable de los parámetros de crecimiento $\mathrm{y}$ aunque ha sido sugerido para especies con una época de desove corta (Defeo et al. 1992a), no sucede en el caso de tilapia, ya que es una especie que presenta múltiples desoves a lo largo de un año (Babiker \& Ibrahim 1979, Morales 1991).

Por otro lado, la aplicación de SLCA en principio podría ser adecuada para estimar los parámetros de crecimiento de $O$. aureus, debido a que los resultados del presente trabajo mostraron que los parámetros de crecimiento se encontraron dentro del intervalo reportado en la literatura por métodos directos. Sin embargo, Jiménez-Badillo (2006), reportó como subestimados los parámetros de crecimiento de $O$. aureus calculados con SLCA, lo cual es acorde con los resultados encontrados en el presente trabajo. SLCA mostró la mayor variabilidad de los estimadores de precisión (valores muy altos de EP y CV), además de que se ha corroborado en estudios previos que SLCA es útil para estimar parámetros de crecimientos en especies longevas con bajas tasas de crecimiento (Basson et al. 1988), caso contrario a lo observado en tilapia que se caracteriza por tener un rápido crecimiento (Gómez-Márquez et al. 2008). Con base en lo anterior y a nuestros resultados, SLCA no es adecuado para evaluar el crecimiento de tilapia, por tanto se sugiere el uso de ELEFAN, debido a su precisión para estimar los parámetros de crecimiento de Oreochromis aureus. Sin embargo, no existe una guía para juzgar en qué casos es apropiado un método en particular (Rosenberg \& Beddington 1987), y por tanto cada especie constituye un caso 
independientemente en cuanto al uso de métodos a emplear para estimar crecimiento. En el caso de $O$. aureus, las diferencias de los parámetros de crecimiento en los distintos cuerpos de agua pueden ser causadas por la temperatura, la disponibilidad del alimento, la actividad metabólica y reproductiva, entre otros factores, lo cual se refleja en $L_{\infty}$ y K (Isaac 1990). Por lo tanto, las estimaciones obtenidas por los tres métodos aplicados aquí, enfatizan que se debe tener cuidado cuando se analiza información que está fuertemente influenciada por procesos ligados a cambios en la longitud, como lo son las condiciones ambientales, la presión de pesca, los patrones de mortalidad, la variabilidad en el reclutamiento, la estacionalidad en el crecimiento, como lo han sugerido Majkowsky \& Hampton (1989).

\section{AGRADECIMIENTOS}

A los pescadores del lago de Chapala por su apoyo brindado durante el registro de datos en campo y a cinco revisores anónimos por sus muy útiles comentarios. Este trabajo forma parte de una investigación realizada en el Centro Regional de Investigación Pesquera en Pátzcuaro, la cual fue financiada por el Instituto Nacional de Pesca.

\section{RESUMEN}

Una de las pesquerías de aguas continentales más importantes en México es la pesca de la tilapia Oreochromis aureus. Con base en el análisis de frecuencia de tallas mediante ELEFAN, SLCA y PROJMAT se estimó el crecimiento en la tilapia en el lago de Chapala, Jalisco, para lo cual se utilizaron 1973 individuos que fueron recolectados de la captura comercial de enero a diciembre 2010. Se cuantificó la variabilidad de los estimadores y el efecto de las muestras mensuales a través de la técnica de remuestreo jackknife. Los parámetros de crecimiento de $L_{\infty}$ y K se unificaron para efectos comparativos mediante el índice de crecimiento estándar ( $\left.\phi^{\prime}\right)$. Con los tres métodos utilizados se estimaron valores de los parámetros de crecimiento que se encuentran dentro del rango reportado en la literatura. La aplicación de jackknife mostró que ELEFAN estimó los mejores resultados, con los valores más bajos en el error porcentual y coeficiente de variación para $L_{\infty}$, en tanto PROJMAT presentó los valores más bajos en los estimadores de precisión para $\mathrm{K}$, la cual fue muy similar a la de
ELEFAN. Los intervalos del índice comparativo de crecimiento $\phi$ ' fueron muy similares a los reportados para la especie en diferentes embalses. Se sugiere el uso de ELEFAN en lugar de SLCA, debido a su precisión para estimar los parámetros de crecimiento de Oreochromis aureus.

Palabras clave: crecimiento, métodos indirectos, jackknife, lago de Chapala, Oreochromis aureus.

\section{REFERENCIAS}

Arredondo-Figueroa, J.L. \& M. Guzmán-Arroyo. 1986. Actual situación taxonómica de las especies de la Tribu Tilapiini (Pisces: Cichlidae) introducidas en México. An. Inst. Biol. UNAM. Ser. Zool. 56: 555-572.

Arredondo-Figueroa, J.L. \& M. Tejeda-Salinas. 1989. El hueso Faríngeo, una estructura útil para la identificación de especies de la tribu Tilapini (Pisces: Cichlidae) introducida en México. An. Inst. Biol. UNAM. Ser. Zool. 16: 58-68.

Babiker, M. \& H. Ibrahim. 1979. Studies on the biology of reproduction in the Cichlid Tilapia nilotica (Linneo): gonadal maturation and fecundity. J. Fish Biol. 14: 437-448.

Basson, M., A.A. Ronsenberg \& J.R. Beddington. 1988. The accuracy and reliability of two new methods for estimating growth parameters from length frequency data. J. Cons. Int. Explor. Mer. 44: 277-285.

Beltrán-Álvarez, R., J. Sánchez-Palacios \& G.L. Valdez. 2010. Edad y crecimiento de la mojarra Oreochromis aureus (Pisces: Cichlidae) en la Presa Sanalona, Sinaloa, México. Rev. Biol. Trop. 58: 325-338.

Bernal, B.F. 1984. Análisis de los factores relacionados con la producción pesquera de Tilapia nilotica en la presa Adolfo López Mateos (Infiernillo), MichoacánGuerrero. Tesis de Licenciatura, Universidad Nacional Autónoma de México, México.

CONAPESCA. 2010. Anuario estadístico de acuacultura y pesca. Comisión Nacional de Acuacultura y Pesca, México.

Defeo, O., F. Arreguín-Sánchez \& J. Sánchez. 1992a. Growth study of the yellow clam Mesodesma mactroides: a comparative analysis of three length-based methods. Sci. Mar. 56: 53-59.

Defeo, O., E. Ortiz \& J.C. Castilla. 1992b. Growth, mortality and recruitment of the yellow clam Mesodesma mactroides in Uruguayan beaches. Mar. Biol. 114: 429-437.

Diario Oficial de la Federación. 2006. Carta Nacional Pesquera, SAGARPA, CONAPESCA Diario Oficial de la Federación del 25 de agosto de 2006, Segunda Sección, México. 
Efron, B. 1982. The Jackknife, the Bootstrap and Other Resampling Plans. Society for Industrial and Applied Mathematics, Filadelfia, Pensilvania, EE.UU.

Galindo, C.G. 2005. Evaluación de tres métodos para estimación del crecimiento basados en el análisis de distribuciones de frecuencia de longitudes. Tesis de Maestría, Instituto Politécnico Nacional, Centro Interdisciplinario de Ciencias Marinas, La Paz, B. C. S., México.

Gayanilo, F.C., P. Sparre \& D. Pauly. 1994. The FAOICLARM Stock Assessment Tools (FISAT) User>s Guide. FAO Comp. Inf. Ser. Fisheries 7, FAO, Roma, Italia.

Gómez-Márquez, J.L., B. Peña-Mendoza, H.I. SalgadoUgarte \& J.L. Arredondo-Figueroa. 2008. Age and growth of tilapia, Oreochromis niloticus (Perciformes: Cichlidae) from a tropical shallow lake in Mexico. Rev. Biol. Trop. 56: 875-884.

Gómez-Ponce, M.A., K. Granados-Flores, C. Padilla, M. López-Hernández \& G. Nuñez-Nogueira. 2011. Edad y crecimiento del híbrido de tilapia Oreochromis niloticus $x$ Oreochromis aureus (Perciformes: Cichlidae) en la represa “Zimapán” Hidalgo, México. Rev. Biol. Trop. 59: 761-770.

Granado, L.C. 1996. Ecología de peces. Universidad de Sevilla, Sevilla, España.

Guzmán-Arroyo, M. 1995. El lago de Chapala, p. 129-145. In G. De la Lanza-Espino \& J.L. García-Calderón (eds.). Lagos y Presas de México. Centro de Ecología y Desarrollo, México, D.F., México.

Haddon, M. 2001. Modelling and quantitative methods in fisheries. Chapman, Florida, EE.UU.

Hernández-Montaño, D. 2006. Lago de Chapala, p. $367-$ 388. In F. Arreguín-Sánchez, L. Bélendez Moreno, I. Méndez Gómez-Humaran, R. Solana Sansores \& C. Rangel Dávalos (eds.). Sustentabilidad y pesca responsable en México. Evaluación y Manejo. Instituto Nacional de la Pesca, México, D.F., México.

Isaac, V.J. 1990. The accuracy of some length-based methods for fish population studies. ICLARM. Tech. Rep. 27, Manila, Filipinas.

Jiménez-Badillo, L. 2006. Age-growth models for tilapia Oreochromis aureus (Perciformes, Cichlidae) of the Infiernillo reservoir, México and reproductive behavior. Rev. Biol. Trop. 54: 577-588.

Jones, J.C., M. Basson \& S. Holden. 1990. LFDA (Length Frequency Analysis Data). A prototype software package for the estimation of growth parameters from length frequency distribution. User's manual (version 2.0). Ren. Res. Ass. Group. Imperial College. Londres, Inglaterra.

Leonce-Valencia, C. \& O. Defeo. 1997. Evaluation of three length-based methods for estimating growth in tropical fishes: the red snapper Lutjanus campechanus of the Campeche Bank (Mexico). Sci. Mar. 61: 297-303

Leslie, P.H. 1945. The use of matrices in certain populations mathematics. Biometrika 35: 213-245.

Levi, D., M.G. Andreoli \& L. Canizzarro. 1987. Use of ELEFAN I for sampling design, p. 311-319. In D. Pauly \& G. P. Morgan (eds.). Length-based Methods in Fisheries Research. ICLARM Conf. Proc. 13, Manila, Filipinas.

Majkowsky, J. \& J. Hampton. 1989. Length-based method for stock assessment and their robustness: a review with emphasis on some Australian experiences, p. 267-278. In E. Chavez (ed.). Proceedings of the Australia-Mexico Workshop on Marine Sciences, México.

Morales, D.A. 1991. La tilapia en México biología, cultivo y pesquerías. AGT, México, D. F., México.

Murphy, B.R., D. Willis \& T.A. Springer. 1991. The relative weight index in fisheries management: Status and needs. Fisheries 16: 30-38.

Nepita-Villanueva, R. \& O. Defeo. 2001. Crecimiento del pulpo Octopus maya (Mollusca: Cephalopoda) de la costa de Yucatán, México: un análisis de largo plazo. Rev. Biol. Trop. 49: 93-101.

Pauly, D. 1984. Fish population dynamics in tropical waters: A manual for use with programmable calculators. ICLARM. Studies and Reviews 8, Manila, Filipinas.

Pauly, D. \& J.L. Munro. 1984. Once more on the comparison of growth in fish and invertebrates. Fishbyte 2: 21 .

Peña-Messina, E., R. Tapia, J.I. Velázquez, A. OrbeMendoza \& J.M. Ruiz. 2010. Growth, mortality and reproduction of the blue tilapia Oreochromis aureus (Perciformes:Cichlidae) in the Aguamilpa Reservoir, Mexico. Rev. Biol. Trop. 58: 1577-1586.

Ramos-Cruz, C. 1995. Reproducción y crecimiento de la mojarra tilapia (Oreochromis aureus) en la presa Benito Juárez, Oaxaca, México. Cien. Pesquera 11: 54-61.

Ramírez, T.J.H. 2007. Edad y crecimiento de la mojarra Oreochromis aureus (Steindachner, 1864), de la Presa Adolfo López Mateos "El Varejonal" Badiraguato Sinaloa, México. Tesis de Licenciatura, Universidad Autónoma de Sinaloa, México.

Ricker, E.W. 1975. Computation and interpretation of biological statistics of fish populations. Bull. Fish. Res. Board. Can. 191: 145-157.

Rosenberg, A.A. \& J.R. Beddington. 1987. Monte Carlo testing of two methods for estimating growth from length frequency data with general conditions for their applicability, p. 283-290. In D. Pauly \& G.P. 
Morgan (eds.). Length-based Methods in Fisheries Research. ICLARM Conf. Proc. 13, Manila, Filipinas.

Rosenberg, A.A., J.R. Beddington \& M. Basson. 1986. The growth and longevity of krill during the first decade of pelagic whaling. Nature 324: 152-154.

Shepherd, J.G. 1987a. A weakly parametric method for the analysis of length composition data, p. 113-119. In D. Pauly \& G.P. Morgan (eds.). Length-based Methods in Fisheries Research. ICLARM Conf. Proc. 13, Manila, Filipinas.
Shepherd, J.G. 1987b. Towards a method for short-term forecasting of catch rates based on length composition, p. 167-176. In D. Pauly \& G.P. Morgan. Lengthbased Methods in Fisheries Research. ICLARM Conf. Proc. 13, Manila, Filipinas.

Sparre, P., E. Ursin \& S.C. Venema. 1989. Introduction to tropical fish stock assessment. Part I. Manual FAO Fish. Tech. Pap. 306.

Trewavas, E. 1983. Tilapiine fishes of the genera Sarotherodon, Oreochromis and Danakilia. British Museum of Natural History, Londres, Inglaterra. 